what happens when the DNA of one mitochondrion is altered and the DNA of another mitochondrion in the same cell remains unaltered?

T. S. WORK

\section{HORMONE INTERACTION}

\section{The Investigation of Hypothalamic-Pituitary-Adrenal Function}

(Proceedings of a Symposium sponsored jointly by Ciba, Horsham, the Society for Endocrinology, and the Endoorine Section of the Royal Society of Medicine, held at the Royal Society of Medicine, London, February 22 and 23, 1967. Memoirs of the Society for Endocrinology, No. 17.) Edited by V. H. T. James and J. Landon. Pp. xii +311. (London: Cambridge University Press, 1968.) 70s. net; $\$ 13.50$.

THE papers constituting this book cover a wide range of clinical and animal studies by experts in the field of hypothalamus-pituitary-adrenal function. On the clinical side, screening tests for pituitary and adrenal insufficiency in adults and children, effects of corticosteroid or adrenocorticotrophin (ACTH) therapy and the pharmacological effect of drugs are assessed. Anatomical and biochemical studies into the actions and control of ACTH and adrenocortical secretion, and effects of stress on the release of ACTH in man and in animals, are investigated. In animal studies hypothalamic control of the secretion of ACTH and "feed-back" mechanism theories are discussed. The corticotrophin activity of synthetic peptide sequences related to ACTH and the influence of vasopressin on pituitary-adrenal activity are described.

In the human foetus there is a "feed-back" mechanism between the pituitary and adrenal glands. Soon after birth and again at the onset of puberty the adrenal cortex changes its cortisol/androgen secretion ratio. The difference in response to administered ACTH in children and adults indicates that there is a pituitary hormone acting synergistically with ACTH to stimulate the adrenal cortex to produce androgen.

There is some evidence from animal studies that neurones in the hypothalamus secrete corticotrophin releasing factor (CRF), the secretion varying in response to the nature of the stimulus. There is probably a site in the median eminence which is sensitive to the varying concentrations of circulating blood cortical steroids. Some similar responses to stress are observed in animals and in man. Varying concentrations of corticosteroids in the blood appear to regulate the rate at which ACTH is synthesized and stored in the pituitary gland, rather than controlling its release. In response to some types of stress ACTH can be released from the gland in spite of a high concentration of circulating corticosteroids. This suggests that stress provides a stimulus for the release of CRF from the hypothalamus which would in turn cause the release of corticotrophin from the anterior pituitary. In rats, chronic stress (for example, handling) leads to an increase in the release of ACTH and thyrotrophin (TSH), while acute stress leads to the release of ACTH and the inhibition of TSH.

In man, stress (which causes the liberation of ACTH) does not produce the same morphological changes in the adrenal cortex as does the injection of ACTH. The administration of ACTH seems to have two actions: (i) it leads to a rapid increase in adrenal blood flow, cortisol secretion and cortisol/corticosterone ratio, and (ii) it has a slow, trophic action in increasing the growth of the gland and causing an increase in RNA and a depletion of lipid. Corticotrophin, however, is a species specific polypeptide and the ACTH preparations in use are of animal origin and may well not act in the same way as the endogenous hormone.

Hypothalamic-pituitary-adrenal function is one of the most difficult fields of endocrine research. In animal work it is difficult to establish good controls, for even handling produces a stress effect. Much of the present knowledge has been gained as a result of animal experiments, but there are considerable species differences which make some results difficult to relate to the findings in man. So far, none of the releasing factors has been completely isolated and until pure CRF is obtainable some of the experimental work must be treated with caution.

May Reed

\section{FORENSIC MEDICINE}

\section{Gradwohl's Legal Medicine}

Edited by Francis E. Camps. Second edition, largely rewritten, with many new illustrations. Pp. xxvii +740 . (Bristol: John Wright and Sons, Ltd., 1968.) 175s. net. The first edition of the American "Gradwohl" which appeared some fifteen years ago was a compilation of monographs rather than a textbook: its only competitor was the much respected "Gonzales, Vance and Helpern", now Helpern (and Umberger), the standard American textbook. It seemed that Gradwohl might die with its original editor; but Francis Camps, a close friend, has revived it-well, rewritten it-with a larger, largely different group of thirty-eight contributors and the assistance of his departmental lecturers. This is a new book: the jacket says "the text has been almost completely re-written and re-designed in order to stress the more objective and critical approach" (shades of Gradwohl).

This new "Gradwohl" now contains nothing of the original fabric. It is a new book, edited by an Englishman with an Anglo-American team of thirty-eight contributors and produced splendidly by John Wright of Bristol. It sets its feet in the statutory and practice ground of the United States as much as in English soil, and bears the "grim portrait of Mathieu Orfila", the nineteenth century French toxicologist, as its frontispiece. The jacket flap says that "the whole text will no doubt help many newly independent countries to formulate a medico-legal system"-a claim that those who know South Africa, or Morocco or India, may dispute.

But let us not deny that this is a stimulating (and sometimes infuriating) book. It is well produced on excellent paper, carries a very large number of illustrations and graphs, and covers a wide field not only of forensic medicine but of science; it even dilates on electric light filaments and the stretchability of knitwear.

Anyone who has tried to marshal nearly forty contributors into a single text will know that the main difficulty is to get them all first to write, then to write with any regard for each other's contributions, and finally to complete their manuscripts by a given date. The bibliography tells the tale: very little of the past five years' literature appears in this text: only single authorship can master this problem and we cannot blame an editor for classical editorial shortcomings.

So the benzidine test, discarded on the advice of the Home Office Scientific Advisory Council (of which the editor is a member), is still warmly recommended, Pugh's extensive writings on cold receive no mention by Mant, 'Brietal' does not achieve mention among Lucas's anaesthetics, and all new law-on abortion, on alcohol, on dangerous drugs and their misuse, the Brain recommendations, and so on-makes no appearance in the text.

Is this book intended for the medical jurist, the medicolegal pathologist, the toxicologist, the forensic scientist? It has something for all. It covers a remarkably wide field from statistical method, ambitiously attempted in two pages by MeDonald of the Police Research Branch, to sexual asphyxias by Brittain. Some new concepts of approach are interesting. 